\title{
Pacific
}

Journal of

Mathematics

\section{CONNECTION RELATIONS AND EXPANSIONS}

Mourad E. H. ISMAIL AND MiZAN RAHMAN

Volume 252 No. 2

August 2011 


\title{
CONNECTION RELATIONS AND EXPANSIONS
}

\author{
Mourad E. H. Ismail AND Mizan RAHMAN
}

\begin{abstract}
We give new proofs of the evaluation of the connection relation for the Askey-Wilson polynomials and for expressing the Askey-Wilson basis in those polynomials using $q$-Taylor series. This led to some inverse relations. We also evaluate the coefficients in the expansions of $(x+b)^{n}$ in various $q$-orthogonal polynomials, including the Askey-Wilson polynomials, which leads to explicit expressions for the moments of the Askey-Wilson weight function. We generalize the $q$-plane wave expansion by expanding $\mathscr{E}_{q}(x ; \alpha)$ in Askey-Wilson polynomials. Further, we prove a bibasic extension of the Nassrallah-Rahman integral and establish a recently conjectured identity of George Andrews.
\end{abstract}

\section{Introduction}

Richard Askey and James Wilson introduced the polynomials that bear their names in their memoir [1985], where they derived, among other properties, the connection relation between Askey-Wilson polynomials with different parameters. One fundamental result of theirs is the evaluation of the Askey-Wilson $q$-beta integral,

$$
\int_{0}^{\pi} \frac{\left(e^{2 i \theta}, e^{-2 i \theta} ; q\right)_{\infty}}{\prod_{j=1}^{4}\left(t_{j} e^{i \theta}, t_{j} e^{-i \theta} ; q\right)_{\infty}} d \theta=\frac{2 \pi\left(t_{1} t_{2} t_{3} t_{4} ; q\right)_{\infty}}{(q ; q)_{\infty} \prod_{1 \leq j<k \leq 4}\left(t_{j} t_{k} ; q\right)_{\infty}} .
$$

All this work was done in the late 1970s and the results were made available to researchers in the area, but the writing took a long time. In the mean time, Nassrallah and Rahman [1985] generalized the Askey-Wilson integral to

$$
\begin{aligned}
\int_{0}^{\pi} \frac{\left(e^{2 i \theta}, e^{-2 i \theta} ; q\right)_{\infty}\left(t_{6} e^{i \theta}, t_{6} e^{-i \theta} ; q\right)_{\infty}}{\prod_{j=1}^{5}\left(t_{j} e^{i \theta}, t_{j} e^{-i \theta} ; q\right)_{\infty}} d \theta & \\
= & \frac{2 \pi\left(t_{1} t_{2} t_{3} t_{4} t_{5} / t_{6} ; q\right)_{\infty} \prod_{j=1}^{5}\left(t_{j} t_{6} ; q\right)_{\infty}}{\left(q, t_{6}^{2} ; q\right)_{\infty} \prod_{1 \leq j<k \leq 5}\left(t_{j} t_{k} ; q\right)_{\infty}} \\
& \quad \times{ }_{8} W_{7}\left(t_{6}^{2} / q ; t_{6} / t_{1}, t_{6} / t_{2}, t_{6} / t_{3}, t_{6} / t_{4}, t_{6} / t_{5} ; q, t_{1} t_{2} t_{3} t_{4} t_{5} / t_{6}\right) .
\end{aligned}
$$

MSC2000: primary 05A19, 33D15; secondary 33D70.

Keywords: connection relations, bibasic integrals, moments of the Askey-Wilson and $q$-ultraspherical distributions, $q$-plane wave expansions, bibasic integrals, Andrews conjecture. 
Remark. The preceding equation is (6.3.9) in [Gasper and Rahman 2004]. As in that reference and in [Ismail 2009], we follow the notation of [Andrews et al. 1999] for $q$-shifted factorials and basic hypergeometric series, and that of [Koekoek and Swarttouw 1998] for orthogonal polynomials.

The Askey-Wilson and Nassrallah-Rahman integrals play a fundamental role in the derivation of the results of this article, which is laid out as follows. Section 2 contains many of the formulas needed, other than (1-1) and (1-2). In particular, the Askey-Wilson polynomials are defined in (2-14).

In Section 3, we first solve the connection-coefficient problem of expanding an Askey-Wilson basis element

$$
\left(a e^{i \theta}, a e^{-i \theta} ; q\right)_{n}
$$

in Askey-Wilson polynomials. The proof utilizes the $q$-integration by parts technique of [Brown et al. 1996]. One application of this expansion is to give a new derivation of a $q$-analogue of the plane wave expansion [Ismail 2009, (4.8.3)]

$$
e^{i x y}=(2 / y)^{v} \Gamma(v) \sum_{n=0}^{\infty}(n+v) i^{n} J_{n+v}(y) C_{n}^{v}(x),
$$

a result first proved in [Ismail and Zhang 1994]. More importantly, we generalize the $q$-plane wave expansion to expand the Ismail-Zhang $q$-exponential function $\mathscr{E}_{q}(x ; \alpha)$ in Askey-Wilson polynomials, which is a new result. The aforementioned connection-coefficient problem is also used to give a new proof of the connection relation of the Askey-Wilson polynomials. Each connection relation may be used to discover an inverse relation of the form $y_{n}=\sum_{k=0}^{n} Y_{n, k} x_{k}$ if and only if $x_{n}=\sum_{k=1}^{n} X_{n, k} y_{k}$. Inverse relations play a fundamental role in combinatorialenumeration problems, as discussed in Riordan's classic [1968]. In the 1970s, interpretations of inverse relations involving $q$-shifted factorials and $q$-binomial coefficients were shown to be instances of Möbius inversion [Rota 1964] and of counting problems involving vector spaces over a finite field [Goldman and Rota 1970]. More recently, very general inverse relations were derived in [Krattenthaler 1989, 1996; Krattenthaler and Schlosser 1999].

Section 4 contains expansions of $x^{n}$ and $(1 \pm x)^{\rho}$ in $q$-ultraspherical polynomials.

Section 5 contains the evaluation of two bibasic integrals which extend the Nassrallah-Rahman integral. They are stated as Theorems 5.1 and 5.2; the latter contains as a special case the evaluation of the moments of the Askey-Wilson weight function. [Corteel and Williams 2007] recently found a beautiful combinatorial expression for the $n$-th moment of the Askey-Wilson measure; this is also part of the results announced in [Corteel and Williams 2010]. Our analytic expression of the moments of the Askey-Wilson weight function is a double sum. 
George Andrews [2011] studied identities involving the Catalan numbers he introduced in [Andrews 1987]. One of his identities was motivated by earlier work of L. Shapiro. Andrews' investigations led him to two summation theorems. One summation theorem is

$$
{ }_{4} \phi_{3}\left(\begin{array}{c}
q^{-2 n}, a, b, q^{1-2 n} / a b \\
q^{2-2 n} / a, q^{2-2 n} / b, q a b
\end{array} \mid q^{2}, q^{2}\right)=\frac{q^{-n}(a, b,-q ; q)_{n}\left(a b ; q^{2}\right)_{n}}{(a b ; q)_{n}\left(a, b ; q^{2}\right)_{n}},
$$

which he proved. He conjectured the validity of the other summation theorem,

$$
\begin{aligned}
{ }_{4} \phi_{3}\left(\begin{array}{c}
q^{-2 n}, a, b, q^{3-2 n} / a b \\
q^{2-2 n} / a, q^{2-2 n} / b, q a b
\end{array} \mid q^{2}, q^{2}\right) \\
=\frac{q^{-n}(a, b / q,-q ; q)_{n}(q-a b)}{(1-b / q)\left(a b-q^{2}\right)\left(1-a b q^{2 n-1}\right)} \\
\quad \times \frac{\left(a b / q^{2} ; q^{2}\right)_{n}}{(a b ; q)_{n}\left(a, b / q^{2} ; q^{2}\right)_{n}}\left(a b q^{2 n-2}\left(q^{2}-b\right)+a b q^{n-1}(1-q)+b-q\right) .
\end{aligned}
$$

Andrews verified (1-5) for $1 \leq n \leq 6$. In Section 6, we give basic hypergeometricseries proofs of both (1-4) and the conjectured identity (1-5). We show that both (1-4) and (1-5) follow from a limiting case of the ${ }_{5} \phi_{4}$ to ${ }_{12} \phi_{11}$ transformation stated in [Gasper and Rahman 2004, (2.8.4)].

\section{Preliminaries}

The expansions of $x^{n}$ and $(1-x)^{\rho}$ in ultraspherical polynomials are

$$
\frac{(2 x)^{n}}{n !}=\sum_{k=0}^{\lfloor n / 2\rfloor} \frac{v+n-2 k}{k !(v)_{n+1-k}} C_{n-2 k}^{v}(x)
$$

[Rainville 1960, (36), p. 283], and

$$
(1-x)^{\rho}=\Gamma(v) \Gamma(v+\rho+1 / 2) \frac{2^{2 v+\rho}}{\sqrt{\pi}} \sum_{k=0}^{\infty} \frac{(k+v)(-\rho)_{k}}{\Gamma(k+2 v+\rho+1)} C_{k}^{v}(x),
$$

valid for $-1<x<1,-\rho<\frac{1}{2}(v+1)$ if $v \geq 0$, and $-\rho<v+\frac{1}{2}$ if $-\frac{1}{2}<v \leq 0$ [Erdélyi et al. 1953, (10.20.6)]. The Chebyshev polynomials are the special cases

$$
T_{n}(x)=\lim _{v \rightarrow 0} \frac{n+2 v}{2 v} C_{n}^{v}(x) \text { and } \quad U_{n}(x)=C_{n}^{1}(x) .
$$

The Chebyshev polynomials are also special cases of the continuous $q$-ultraspherical polynomials, since

$$
T_{n}(x)=\lim _{\beta \rightarrow 1} \frac{1-\beta q^{n}}{1-\beta^{2}} C_{n}^{\nu}(x ; \beta \mid q) \quad \text { and } \quad U_{n}(x)=C_{n}(x ; q \mid q) .
$$


The Rogers connection relation for the $q$-ultraspherical polynomials is

$$
C_{n}(x ; \gamma \mid q)=\sum_{k=0}^{\lfloor n / 2\rfloor} \frac{\beta^{k}(\gamma / \beta ; q)_{k}(\gamma ; q)_{n-k}}{(q ; q)_{k}(q \beta ; q)_{n-k}} \frac{1-\beta q^{n-2 k}}{1-\beta} C_{n-2 k}(x ; \beta \mid q)
$$

[Ismail 2009, (13.3.1)]. The Ismail-Zhang $q$-exponential function is

$$
\begin{aligned}
& \mathscr{E}_{q}(\cos \theta ; \alpha) \\
& \quad=\frac{\left(\alpha^{2} ; q^{2}\right)_{\infty}}{\left(q \alpha^{2} ; q^{2}\right)_{\infty}} \sum_{n=0}^{\infty}\left(-i e^{i \theta} q^{(1-n) / 2},-i e^{-i \theta} q^{(1-n) / 2} ; q\right)_{n} \frac{(-i \alpha)^{n}}{(q ; q)_{n}} q^{n^{2} / 4}
\end{aligned}
$$

[Ismail 2009, §14.1].

We shall always use the notation

$$
x=\cos \theta, \quad z=e^{i \theta}, \quad f(x)=\breve{f}(z) .
$$

The set of polynomials $\left\{\left(a e^{i \theta}, a e^{-i \theta} ; q\right)_{n}: n=0,1, \ldots\right\}$ is a basis for the space of all polynomials, and is called the Askey-Wilson basis. The connection formula for the Askey-Wilson basis is

$$
\frac{\left(b e^{i \theta}, b e^{-i \theta} ; q\right)_{n}}{(q, a b ; q)_{n}}=\sum_{k=0}^{n} \frac{\left(a e^{i \theta}, a e^{-i \theta} ; q\right)_{k}}{(q, a b ; q)_{k}} \frac{(b / a ; q)_{n-k}}{(q ; q)_{n-k}}\left(\frac{b}{a}\right)^{k}
$$

[Ismail 1995]; see also the proof of Theorem 12.2.3 in [Ismail 2009].

We recall the definition of the Askey-Wilson operator,

$$
\left(\mathscr{D}_{q} f\right)(x)=\frac{\breve{f}\left(q^{1 / 2} z\right)-\breve{f}\left(q^{-1 / 2} z\right)}{\left(q^{1 / 2}-q^{-1 / 2}\right)(z-1 / z) / 2} .
$$

It is easy to see that

$$
\mathscr{D}_{q}\left(a e^{i \theta}, a e^{-i \theta} ; q\right)_{n}=-\frac{2 a\left(1-q^{n}\right)}{1-q}\left(a q^{1 / 2} e^{i \theta}, a q^{1 / 2} e^{-i \theta} ; q\right)_{n-1}
$$

[Ismail 2009, (12.2.2)]. We shall use the inner product

$$
\langle f, g\rangle:=\int_{-1}^{1} f(x) \overline{g(x)} \frac{d x}{\sqrt{1-x^{2}}} .
$$

Let

$$
H_{v}:=\left\{f: f((z+1 / z) / 2) \text { is analytic for } q^{v} \leq|z| \leq q^{-v}\right\} .
$$

The following theorem - an analogue of integration by parts - is due to Brown, Evans and Ismail [Brown et al. 1996]; see also [Ismail 2009, §16.1]. 
Theorem 2.1. The Askey-Wilson operator $\mathscr{D}_{q}$ satisfies, for $f, g \in H_{1 / 2}$,

$$
\begin{array}{r}
\left\langle\mathscr{D}_{q} f, g\right\rangle=\frac{\pi \sqrt{q}}{1-q}\left[f\left(\frac{q^{1 / 2}+q^{-1 / 2}}{2}\right) \overline{g(1)}-f\left(-\frac{q^{1 / 2}+q^{-1 / 2}}{2}\right) \overline{g(-1)}\right] \\
-\left\langle f, \sqrt{1-x^{2}} \mathscr{D}_{q}\left(g(x)\left(1-x^{2}\right)^{-1 / 2}\right)\right\rangle .
\end{array}
$$

The Askey-Wilson polynomials have the basic hypergeometric representation

$(2-14) \quad p_{n}(x ; \mathbf{t} \mid q)$

$$
=t_{1}^{-n}\left(t_{1} t_{2}, t_{1} t_{3}, t_{1} t_{4} ; q\right)_{n}{ }_{4} \phi_{3}\left(\begin{array}{c|c}
q^{-n}, t_{1} t_{2} t_{3} t_{4} q^{n-1}, t_{1} e^{i \theta}, t_{1} e^{-i \theta} \\
t_{1} t_{2}, t_{1} t_{3}, t_{1} t_{4}
\end{array} \mid q, q\right),
$$

where $\mathbf{t}$ stands for the ordered quadruple $\left(t_{1}, t_{2}, t_{3}, t_{4}\right)$. Their weight function is

$$
w(x, \mathbf{t} \mid q)=\frac{\left(e^{2 i \theta}, e^{-2 i \theta} ; q\right)_{\infty}}{\prod_{j=1}^{4}\left(t_{j} e^{i \theta}, t_{j} e^{-i \theta} ; q\right)_{\infty}} \frac{1}{\sqrt{1-x^{2}}}, \quad x=\cos \theta \in(-1,1),
$$

The Askey-Wilson polynomials satisfy the orthogonality relation

$$
\begin{aligned}
\int_{-1}^{1} p_{m}(x ; \mathbf{t} \mid q) p_{n}(x ; \mathbf{t} \mid q) & w(x ; \mathbf{t} \mid q) d x \\
& =h_{n}(\mathbf{t}) \delta_{m, n} \\
& =\frac{2 \pi\left(t_{1} t_{2} t_{3} t_{4} q^{2 n} ; q\right)_{\infty}\left(t_{1} t_{2} t_{3} t_{4} q^{n-1} ; q\right)_{n}}{\left(q^{n+1} ; q\right)_{\infty} \prod_{1 \leq j<k \leq 4}\left(t_{j} t_{k} q^{n} ; q\right)_{\infty}} \delta_{m, n},
\end{aligned}
$$

for $\max \left\{\left|t_{1}\right|,\left|t_{2}\right|,\left|t_{3}\right|,\left|t_{4}\right|\right\}<1$. The Askey-Wilson polynomials also satisfy the Rodrigues-type formula

$$
w(x ; \mathbf{t} \mid q) p_{n}(x ; \mathbf{t} \mid q)=\left(\frac{q-1}{2}\right)^{n} q^{n(n-1) / 4} \mathscr{D}_{q}^{n} w\left(x ; q^{n / 2} \mathbf{t} \mid q\right) .
$$

The Chebyshev polynomials are also special Askey-Wilson polynomials; indeed,

$$
\begin{aligned}
& p_{n}(x ; q,-q, \sqrt{q},-\sqrt{q} \mid q)=\left(q^{n+2} ; q\right)_{n} U_{n}(x), \\
& p_{0}(x ; \mathbf{t} \mid q)=T_{0}(x)=1, \\
& p_{n}(x ; 1,-1, \sqrt{q},-\sqrt{q} \mid q)=2\left(q^{n} ; q\right)_{n} T_{n}(x) \quad \text { for } n>0 .
\end{aligned}
$$

We shall also use the $q$-Taylor expansion stated next.

Theorem 2.2 [Ismail 1995]. Let

$$
x_{n}=\left(a q^{n / 2}+q^{-n / 2} / a\right) / 2 \text { for } 0<q<1,0<a<1,
$$


If $f(x)$ is a polynomial, then

with

$$
f(x)=\sum_{k=0}^{\infty} f_{k}\left(a e^{i \theta}, a e^{-i \theta} ; q\right)_{k},
$$

$$
f_{k}=\frac{(q-1)^{k}}{(2 a)^{k}(q ; q)_{k}} q^{-k(k-1) / 4}\left(\mathscr{D}_{q}^{k} f\right)\left(x_{k}\right) .
$$

For a proof and details, see [Ismail 2009, Theorem 12.2.2].

\section{Connection formulas and expansions}

Lemma 3.1. We have the integral evaluation

$$
\begin{aligned}
& \int_{-1}^{1}\left(a e^{i \theta}, a e^{-i \theta} ; q\right)_{n} w(x ; \mathbf{t} \mid q) d x= \\
& \quad=\frac{2 \pi\left(t_{1} a, a / t_{1} ; q\right)_{n}\left(t_{1} t_{2} t_{3} t_{4} ; q\right)_{\infty}}{(q ; q)_{\infty} \prod_{1 \leq j<m \leq 4}\left(t_{j} t_{m} ; q\right)_{\infty}} 4 \phi_{3}\left(\begin{array}{c}
q^{-n}, t_{1} t_{2}, t_{1} t_{3}, t_{1} t_{4} \\
t_{1} a, t_{1} t_{2} t_{3} t_{4}, q^{1-n} t_{1} / a
\end{array} \mid q, q\right) .
\end{aligned}
$$

This integral can be evaluated by writing

$$
\left(a e^{i \theta}, a e^{-i \theta} ; q\right)_{n}=\frac{\left(a e^{i \theta}, a e^{-i \theta} ; q\right)_{\infty}}{\left(a q^{n} e^{i \theta}, a q^{n} e^{-i \theta} ; q\right)_{\infty}},
$$

then using the Nassrallah-Rahman integral (1-2) and the Watson transformation [Gasper and Rahman 2004, (III.18)]. It also follows by expanding $\left(a e^{i \theta}, a e^{-i \theta} ; q\right)_{n}$ in $\left\{\left(t_{1} e^{i \theta}, t_{1} e^{-i \theta} ; q\right)_{k}: 0 \leq k \leq n\right\}$ by using (2-8), and then applying the AskeyWilson integral (1-1); see also [Ismail and Stanton 1998, Thm. 3].

Our first result is the next expansion of $\left(b e^{i \theta}, b e^{-i \theta} ; q\right)_{n}$ in Askey-Wilson polynomials.

\section{Theorem 3.2.}

$$
\left(b e^{i \theta}, b e^{-i \theta} ; q\right)_{n}=\sum_{k=0}^{n} f_{n, k}(b, \mathbf{t}) p_{k}(x ; \mathbf{t} \mid q),
$$

where

$$
\begin{aligned}
f_{n, k}(b, \mathbf{t})=\frac{(-b)^{k} q^{\left(\begin{array}{c}
k \\
2
\end{array}\right)(q ; q)_{n}\left(b / t_{4}, b t_{4} q^{k} ; q\right)_{n-k}}}{\left(q, t_{1} t_{2} t_{3} t_{4} q^{k-1} ; q\right)_{k}(q ; q)_{n-k}} \\
\quad \times{ }_{4} \phi_{3}\left(\begin{array}{c}
q^{k-n}, t_{1} t_{4} q^{k}, t_{2} t_{4} q^{k}, t_{3} t_{4} q^{k} \\
b t_{4} q^{k}, t_{1} t_{2} t_{3} t_{4} q^{2 k}, q^{1-n+k} t_{4} / b
\end{array} \mid q, q\right) .
\end{aligned}
$$

Proof. It is clear that

$$
f_{n, k} h_{k}(\mathbf{t})=\left\langle p_{k}(x ; \mathbf{t} \mid q) w(x ; \mathbf{t} \mid q), \sqrt{1-x^{2}}\left(b e^{i \theta}, b e^{-i \theta} ; q\right)_{n}\right\rangle
$$




$$
\begin{aligned}
& =\left(\frac{q-1}{2}\right)^{k} q^{k(k-1) / 4}\left\langle\mathscr{D}_{q}^{k} w\left(x ; q^{k / 2} \mathbf{t} \mid q\right), \sqrt{1-x^{2}}\left(b e^{i \theta}, b e^{-i \theta} ; q\right)_{n}\right\rangle \\
& =\left(\frac{1-q}{2}\right)^{k} q^{k(k-1) / 4} \int_{-1}^{1} w\left(x ; q^{k / 2} \mathbf{t} \mid q\right) \mathscr{D}_{q}^{k}\left(b e^{i \theta}, b e^{-i \theta} ; q\right)_{n} d x \\
& =\frac{(-b)^{k}(q ; q)_{n}}{(q ; q)_{n-k}} q^{\left({ }_{2}^{k}\right)} \int_{-1}^{1}\left(b q^{k / 2} e^{i \theta}, b q^{k / 2} e^{-i \theta} ; q\right)_{n-k} w\left(x ; q^{k / 2} \mathbf{t} \mid q\right) d x .
\end{aligned}
$$

In these steps we used the Rodrigues formula (2-17), as well as (2-13) and (2-10). The result follows from a slight variation of Lemma 3.1.

Our first application of Theorem 3.2 is the connection relation for the AskeyWilson polynomials.

Corollary 3.3. We have the connection relation

$$
p_{n}(x ; \mathbf{b})=\sum_{k=o}^{n} c_{n, k}(\mathbf{b}, \mathbf{a}) p_{k}(x ; \mathbf{a}),
$$

where

$$
\begin{gathered}
c_{n, k}(\mathbf{b}, \mathbf{a})=\frac{b_{4}^{k-n}\left(b_{1} b_{2} b_{3} b_{4} q^{n-1} ; q\right)_{k}\left(q, b_{1} b_{4}, b_{2} b_{4}, b_{3} b_{4} ; q\right)_{n}}{(q ; q)_{n-k}\left(q, a_{1} a_{2} a_{3} a_{4} q^{k-1} ; q\right)_{k}\left(b_{1} b_{4}, b_{2} b_{4}, b_{3} b_{4} ; q\right)_{k}} \\
\times q^{k(k-n)} \sum_{j, l \geq 0} \frac{\left(q^{k-n}, b_{1} b_{2} b_{3} b_{4} q^{n+k-1}, a_{4} b_{4} q^{k} ; q\right)_{j+l} q^{j+l}}{\left(b_{1} b_{4} q^{k}, b_{2} b_{4} q^{k}, b_{3} b_{4} q^{k} ; q\right)_{j+l}(q ; q)_{j}(q ; q)_{l}} \\
\times \frac{\left(a_{1} a_{4} q^{k}, a_{2} a_{4} q^{k}, a_{3} a_{4} q^{k} ; q\right)_{l}\left(b_{4} / a_{4} ; q\right)_{j}}{\left(a_{4} b_{4} q^{k}, a_{1} a_{2} a_{3} a_{4} q^{2 k} ; q\right)_{l}}\left(\frac{b_{4}}{a_{4}}\right)^{l} .
\end{gathered}
$$

Proof. The follows by expanding the left-hand side of (3-4) in the Askey-Wilson basis $\left\{\left(a_{1} e^{i \theta}, a_{1} e^{-i \theta} ; q\right)_{k}\right\}$, then applying Theorem 3.2.

Corollary 3.3 is Theorem 14.4.2 in [Ismail 2009]. When $a_{4}=b_{4}$, the double series in (3-4) reduces to a ${ }_{5} \phi_{4}$ and we get a result of [Askey and Wilson 1985]. See also [Gasper and Rahman 2004, (7.6.2)-(7.6.3)]. For another proof, see [Ismail and Zhang 2005], which also uses (2-13). Note that, in view of the orthogonality relation (2-16), Corollary 3.3 is equivalent to Theorem 3.2.

The special case $b=t_{3}$ of Theorem 3.2 is interesting. The result, after interchanging $t_{1}$ and $t_{3}$, is

$$
\begin{aligned}
& \left(t_{1} e^{i \theta}, t_{1} e^{-i \theta} ; q\right)_{n}=\sum_{k=0}^{n}\left[\begin{array}{l}
n \\
k
\end{array}\right]_{q}\left(-t_{1}\right)^{k} q^{\left(\begin{array}{c}
k \\
2
\end{array}\right)} \frac{\left(t_{1} t_{2}, t_{1} t_{3}, t_{1} t_{4} ; q\right)_{n}}{\left(t_{1} t_{2}, t_{1} t_{3}, t_{1} t_{4} ; q\right)_{k}} \frac{1-t_{1} t_{2} t_{3} t_{4} q^{2 k-1}}{1-t_{1} t_{2} t_{3} t_{4} / q} \\
& \times \frac{\left(t_{1} t_{2} t_{3} t_{4} / q ; q\right)_{k}}{\left(t_{1} t_{2} t_{3} t_{4} ; q\right)_{n+k}} p_{k}(x ; \mathbf{t} \mid q) .
\end{aligned}
$$


Theorem 3.4. The following relations are equivalent:

$$
\begin{aligned}
& B_{n}=\frac{\left(t_{1} t_{2}, t_{1} t_{3}, t_{1} t_{4} ; q\right)_{n}}{t_{1}^{n}} \sum_{k=0}^{n} \frac{\left(q^{-n}, t_{1} t_{2} t_{3} t_{4} q^{n-1} ; q\right)_{k}}{(q ; q)_{k} \prod_{j=2}^{4}\left(t_{1} t_{j} ; q\right)_{k}} q^{k} A_{k} \\
& A_{n}=\sum_{k=0}^{n} \frac{t_{1}^{k} q^{\left(\begin{array}{l}
k \\
2
\end{array}\right)\left(t_{1} / t_{4}, t_{1} t_{2} q^{k}, t_{1} t_{3} q^{k}, t_{1} t_{4} q^{k} ; q\right)_{n-k}}\left(B_{k}, t_{1} t_{2} t_{3} t_{4} q^{k-1} ; q\right)_{k}\left(q, t_{1} t_{2} t_{3} t_{4} q^{2 k} ; q\right)_{n-k}}{B_{k}}
\end{aligned}
$$

Proof. We set $b=t_{1}$ in (3-2) and take (2-14) into account. The ${ }_{4} \phi_{3}$ in (3-3) becomes a ${ }_{3} \phi_{2}$, and can be summed by the $q$-analogue of the Pfaff-Saalschütz theorem.

Theorem 3.4 is known [Krattenthaler 1989; 1996]. An interesting question is to explore where such inverse pair lives from the point of view of the Möbius function on lattices [Rota 1964], because the lattices which will lead to such a deep result will be very interesting. It is also interesting to explore the concept of Bailey pairs [Andrews 1986] from the Möbius-inversion point of view.

The $q$-ultraspherical polynomials are special Askey-Wilson polynomials, since

$$
p_{n}(x ; \sqrt{\beta},-\sqrt{\beta}, \sqrt{q \beta},-\sqrt{q \beta} \mid q)=\frac{\left(q, \beta^{2} q^{n} ; q\right)_{n}}{(\beta ; q)_{n}} C_{n}(x ; \beta \mid q) .
$$

The $q$-plane wave expansion in $q$-ultraspherical polynomials is

$$
\begin{aligned}
\mathscr{E}_{q}(x ; i \alpha)=\frac{(\alpha)^{-v}(q ; q)_{\infty}}{\left(-q \alpha^{2} ; q^{2}\right)_{\infty}\left(q^{v+1} ; q\right)_{\infty}} \sum_{n=0}^{\infty} \frac{\left(1-q^{n+v}\right)}{\left(1-q^{v}\right)} q^{n^{2} / 4} i^{n} \\
\times J_{v+n}^{(2)}(2 \alpha ; q) C_{n}\left(x ; q^{v} \mid q\right) ;
\end{aligned}
$$

see [Ismail and Zhang 1994].

Another application of Theorem 3.2 is this generalization of (3-10):

Theorem 3.5. We have the following generalization of the q-plane wave expansion function:

$$
\begin{aligned}
\mathscr{E}_{q}(x ; \alpha)= & \frac{\left(\alpha^{2} ; q^{2}\right)_{\infty}}{\left(q \alpha^{2} ; q\right)_{\infty}} \sum_{n=0}^{\infty} \frac{\alpha^{n} q^{n^{2} / 4} p_{n}(x ; \mathbf{t})}{\left(q, t_{1} t_{2} t_{3} t_{4} q^{n-1} ; q\right)_{n}} \\
& \times \sum_{k=0}^{\infty} \frac{\left(-\alpha / t_{4}\right)^{k}}{(q ; q)_{k}}\left(-q^{1+n-k} t_{4}^{2} ; q^{2}\right)_{k} q^{k(k-2 n) / 4} \\
& \quad \times{ }_{4} \phi_{3}\left(\begin{array}{c}
q^{-k}, t_{1} t_{4} q^{n}, t_{2} t_{4} q^{n}, t_{3} t_{4} q^{n} \\
-i t_{4} q^{(1-k+n) / 2}, i t_{4} q^{(1-k+n) / 2}, t_{1} t_{2} t_{3} t_{4} q^{2 n}
\end{array} \mid q, q\right) .
\end{aligned}
$$

Proof. Expand the $\mathscr{E}_{q}$ in the Askey-Wilson basis via (2-6), then apply (3-2). 
Another proof of Theorem 3.5. Since $\mathscr{E}_{q}(x ; \alpha) \in L_{2}[-1,1, w(x ; \mathbf{t})]$, we set

$$
\mathscr{E}_{q}(x ; \alpha)=\sum_{n=0}^{\infty} c_{n} p_{n}(x ; \mathbf{t}) .
$$

Using (2-17), the divided-difference relation $\mathscr{D}_{q} \mathscr{\mathscr { E }}_{q}(x ; \alpha)=2 \alpha q^{1 / 4} /(1-q) \mathscr{E}_{q}(x ; \alpha)$ and the $q$-integration by parts (2-13), we find that

$$
\begin{aligned}
c_{n} h_{n}(\mathbf{t})= & \int_{-1}^{1} \mathscr{E}_{q}(x ; \alpha) p_{n}(x ; \mathbf{t}) w(x ; \mathbf{t}) d x \\
= & \left(\frac{q-1}{2}\right)^{n} q^{\left(\begin{array}{c}
n \\
2
\end{array}\right) / 2} \int_{-1}^{1} \mathscr{E}_{q}(x ; \alpha) \mathscr{D}_{q}^{n} w\left(x ; q^{n / 2} \mathbf{t}\right) d x \\
= & \alpha^{n} q^{n^{2} / 4} \int_{-1}^{1} \mathscr{E}_{q}(x ; \alpha) w\left(x ; q^{n / 2} \mathbf{t}\right) d x \\
= & \frac{\left(\alpha^{2} ; q^{2}\right)_{\infty}}{\left(q \alpha^{2} ; q^{2}\right)_{\infty}} \alpha^{n} q^{n^{2} / 4} \sum_{k=0}^{\infty} \frac{(-i \alpha)^{k}}{(q ; q)_{k}} q^{k^{2} / 4} \\
& \quad \times \int_{0}^{\pi} w\left(\cos \theta ; q^{n / 2} \mathbf{t}\right)\left(-i q^{(1-k) / 2} e^{i \theta},-i q^{(1-k) / 2} e^{-i \theta} ; q\right)_{k} \sin \theta d \theta .
\end{aligned}
$$

The integral above is

$$
\begin{aligned}
& 2 \pi\left(-i t_{4} q^{(1+n-k) / 2},-i t_{4} q^{(1-n-k) / 2} / t_{4} ; q\right)_{k}\left(t_{1} t_{2} t_{3} t_{4} q^{2 n} ; q\right)_{\infty} \\
& (q ; q)_{\infty} \prod_{1 \leq j<m \leq 4}\left(t_{j} t_{m} q^{n} ; q\right)_{\infty} \\
& \times{ }_{4} \phi_{3}\left(\begin{array}{c|c}
q^{-k}, t_{1} t_{4} q^{n}, t_{2} t_{4} q^{n}, t_{3} t_{4} q^{n} \\
-i t_{4} q^{(1-k+n) / 2}, i t_{4} q^{(1-k+n) / 2}, t_{1} t_{2} t_{3} t_{4} q^{2 n}
\end{array} \mid q, q\right) .
\end{aligned}
$$

The result now follows from (2-16).

In the case of $q$-ultraspherical polynomials, the ${ }_{4} \phi_{3}$ in (3-11) can be summed by Andrews' $q$-analogue of Watson's ${ }_{3} F_{2}$ sum [Gasper and Rahman 2004, (II.17)]. Thus, the ${ }_{4} \phi_{3}$ is zero for $k$ odd and, when $k$ is replaced by $2 k$, the ${ }_{4} \phi_{3}$ is

$$
\beta^{2 k} q^{2 n k+k} \frac{\left(q,-q^{1-n-2 k} / \beta ; q^{2}\right)_{k}}{\left(-\beta q^{n+2-2 k}, \beta^{2} q^{2 n+2} ; q^{2}\right)_{k}} .
$$

Thus, the $k$-sum in (3-11) is ${ }_{2} \phi_{1}\left(-\beta q^{n+2},-\beta q^{n+1} ; \beta^{2} q^{2 n+2} ; q^{2}, \alpha^{2}\right)$. Therefore,

$$
\begin{aligned}
\mathscr{E}_{q}(x ; \alpha)=\frac{\left(\alpha^{2} ; q^{2}\right)_{\infty}}{\left(q \alpha^{2} ; q\right)_{\infty}} & \sum_{n=0}^{\infty} \frac{\alpha^{n} q^{n^{2} / 4}}{(\beta ; q)_{n}} \\
& \times{ }_{2} \phi_{1}\left(\begin{array}{c}
-\beta q^{n+2},-\beta q^{n+1} \\
\beta^{2} q^{2 n+2}
\end{array} \mid q^{2}, \alpha^{2}\right) C_{n}(x ; \beta \mid q) .
\end{aligned}
$$


By equating the left sides of (3-12) and (3-10), we establish the identity

$$
\begin{aligned}
J_{v}^{(2)}(2 \alpha ; q) & =\frac{\alpha^{\nu}\left(-\alpha^{2} ; q^{2}\right)_{\infty}}{\left(q^{v+1} ; q\right)_{\infty}}{ }_{2} \phi_{1}\left(\begin{array}{c}
-q^{v+2},-q^{v+1} \\
q^{2 v+2}
\end{array} \mid q^{2},-\alpha^{2}\right) \\
& =\frac{\alpha^{\nu}\left(q^{v+1} \alpha^{2} ; q^{2}\right)_{\infty}}{\left(q^{v+1} ; q\right)_{\infty}}{ }_{2} \phi_{2}\left(\begin{array}{c}
-q^{v+2},-q^{v+1} \\
q^{2 v+2}, q^{v+2} \alpha^{2}
\end{array} \mid q^{2}, q^{\nu+1} \alpha^{2}\right),
\end{aligned}
$$

after applying the ${ }_{2} \phi_{1}$ to ${ }_{2} \phi_{2}$ transformation [Gasper and Rahman 2004, (III.4)]. The representation of $J_{v}^{(2)}$ as a ${ }_{2} \phi_{2}$ is due to [Rahman 1987].

The double series in (3-11) also reduces to a single series in the case of continuous $q$-Jacobi polynomials, $t_{2}=t_{1} q^{1 / 2}$ and $t_{4}=t_{3} q^{1 / 2}$, yielding a result in [Ismail et al. 1996]. The details however are not lengthy and will be omitted.

\section{Expansions of $x^{n}$ and $(1 \pm x)^{\rho}$}

Theorem 4.1. The expansion

$$
\begin{aligned}
& (1-x)^{\rho}=\frac{4}{\sqrt{\pi}} 2^{\rho} \Gamma(\rho+3 / 2) \\
& \times \sum_{k=0}^{\infty} \frac{1-\beta q^{k}}{1-\beta}\left(\sum_{j=0}^{\infty} \frac{(k+2 j+1)(-\rho)_{k+2 j} \beta^{j}(q / \beta ; q)_{j}(q ; q)_{k+j}}{(q, q)_{j}(q \beta ; q)_{k+j} \Gamma(k+2 j+\rho+3)}\right) C_{k}(x ; \beta \mid q)
\end{aligned}
$$

holds for $-1<x<1, \rho>-1$ and $\beta \in(0,1)$. The expansion for $(1+x)^{\rho}$ is similar, since $C_{n}(-x ; \beta \mid q)=(-1)^{n} C_{n}(x ; \beta \mid q)$.

Proof. Apply (2-2) with $v=1$, then expand $C_{k}^{1}(x)=U_{k}(x)=C_{k}(x ; q \mid q)$ in $C_{j}(x ; \beta \mid q)$ by using (2-5), then rearrange the series. The expansion (2-2) holds for $\rho>-1$. The rearrangement is valid because the double series in the theorem converges absolutely for $\rho>-1$, in view of the asymptotic formula [Ismail 2009, (13.4.5)] and the well-known fact that $n^{b-a} \Gamma(n+a) / \Gamma(n+b) \rightarrow 1$ as $n \rightarrow+\infty$.

It is interesting to note that, as $q \rightarrow 1$, the expansion (4-1) should reduce to (2-2). Indeed with $\beta=q^{v}$ the $q \rightarrow 1$ limit of the quantity in square brackets is a wellpoised ${ }_{5} F_{4}$ at $x=1$, which can be summed, see Slater [Slater 1966, (III.12)]. So we could have discovered the abovementioned sum if it was not already known.

Theorem 4.2. For nonnegative integers $n$ we have the $q$-ultraspherical expansion

$$
\begin{aligned}
x^{n}=\frac{n !}{2^{n}} \sum_{m=0}^{\lfloor n / 2\rfloor} \frac{1-\beta q^{n-2 m}}{1-\beta} C_{n-2 m}(x ; \beta \mid q) & \\
& \times \sum_{k=0}^{m} \frac{n+1-2 k}{k !(n+1-k) !} \frac{\beta^{m-k}(q / \beta ; q)_{m-k}(q ; q)_{n-m-k}}{(q ; q)_{m-k}(q \beta ; q)_{n-m-k}} .
\end{aligned}
$$


Proof. The expansion (4-2) follows immediately from letting $v=1$ in (2-1) then use (2-5) with $\gamma=1$.

Note that

$$
\frac{n !(n+1-2 k)}{k !(n+1-k) !}=\left(\begin{array}{l}
n \\
k
\end{array}\right)-\left(\begin{array}{c}
n \\
k-1
\end{array}\right) .
$$

With $\beta=q^{v}$, the limit of the $k$-sum in (4-2) as $q \rightarrow 1$ is a very well-poised ${ }_{4} F_{3}$ at $x=-1$, which can be summed [Slater 1966, (III.11)].

\section{Two bibasic integrals}

In this section we give evaluations of the integral (5-2) and the more general integral (5-3). The proof uses the bibasic expansion

$$
\begin{aligned}
& \frac{\left(q, q a^{2} ; q\right)_{\infty}}{\left(q a e^{i \theta}, q a e^{-i \theta} ; q\right)_{\infty}}\left(b e^{i \theta}, b e^{-i \theta} ; p\right)_{\infty} \\
& =\sum_{k=0}^{\infty} \frac{1-a^{2} q^{2 k}}{1-a^{2}} \frac{\left(a^{2}, a e^{i \theta}, a e^{-i \theta} ; q\right)_{k}}{\left(q, q a e^{i \theta}, a q e^{-i \theta} ; q\right)_{k}}(-1)^{k} q^{\left(\begin{array}{c}
k+1 \\
2
\end{array}\right)}\left(a b q^{k}, b q^{-k} / a ; p\right)_{\infty},
\end{aligned}
$$

which is valid for $0<p<q$, or $p=q$ and $|b|<|a|$ [Ismail and Stanton 2003].

Theorem 5.1. We have the bibasic integral evaluation

$$
\begin{aligned}
& \int_{0}^{\pi} \frac{\left(e^{2 i \theta}, e^{-2 i \theta} ; q\right)_{\infty}\left(b e^{i \theta}, b e^{-i \theta} ; p\right)_{\infty}}{\prod_{j=1}^{5}\left(a_{j} e^{i \theta}, a_{j} e^{-i \theta} ; q\right)_{\infty}} d \theta \\
= & \frac{2 \pi\left(a_{2} a_{3} a_{4} a_{5} / q ; q\right)_{\infty}}{(q ; q)_{\infty} \prod_{2 \leq r<s \leq 5}\left(a_{r} a_{s} ; q\right)_{\infty}} \frac{1}{\left(q, q a_{1}^{2} ; q\right)_{\infty}} \\
& \times \sum_{k=0}^{\infty} \frac{1-a_{1}^{2} q^{2 k}}{1-a_{1}^{2}} \frac{\left(a_{1}^{2} ; q\right)_{k}}{(q ; q)_{k}} \frac{1-a_{1}^{2} q^{2 k+1}}{\prod_{s=2}^{5}\left(1-a_{1} a_{s} q^{k}\right)}(-1)^{k} q^{\left({ }^{k+1}\right)}\left(a_{1} b q^{k}, \frac{b q^{-k}}{a_{1}} ; p\right)_{\infty} \\
& \times{ }_{8} W_{7}\left(a_{1}^{2} q^{2 k+1} ; q, q^{k+1} \frac{a_{1}}{a_{2}}, q^{k+1} \frac{a_{1}}{a_{3}}, q^{k+1} \frac{a_{1}}{a_{4}}, q^{k+1} \frac{a_{1}}{a_{5}} ; q, \frac{a_{2} a_{3} a_{4} a_{5}}{q}\right) \\
= & \frac{2 \pi \prod_{j=2}^{5}\left(a_{1} a_{2} a_{3} a_{4} a_{5} / a_{j} ; q\right)_{\infty}}{\left(q, a_{1}^{2} a_{2} a_{3} a_{4} a_{5} ; q\right)_{\infty} \prod_{1 \leq r<s \leq 5}\left(a_{r} a_{s} ; q\right)_{\infty}} \\
& \times \sum_{k=0}^{\infty} \frac{\left(a_{1}^{2} ; q\right)_{k}\left(a_{1}^{2} a_{2} a_{3} a_{4} a_{5} ; q\right)_{2 k}}{(q ; q)_{k}\left(a_{1}^{2} ; q\right)_{2 k}} \\
& \times \prod_{j=2}^{5} \frac{\left(a_{1} a_{j} ; q\right)_{k}}{\left(a_{1} a_{2} a_{3} a_{4} a_{5} / a_{j} ; q\right)_{k}}(-1)^{k} q^{\left({ }_{2}{ }_{2}\right)}\left(a_{1} b q^{k}, \frac{b q^{-k}}{a_{1}} ; p\right)_{\infty} \\
& \times{ }_{8} W_{7}\left(a_{1}^{2} a_{2} a_{3} a_{4} a_{5} q^{2 k-1} ; a_{1} a_{2} q^{k}, a_{1} a_{3} q^{k}, a_{1} a_{4} q^{k}, a_{1} a_{5} q^{k}, \frac{a_{2} a_{3} a_{4} a_{5}}{q} ; q, q\right) .
\end{aligned}
$$


Proof. In view of (5-1), the left-hand side of (5-2) is

$$
\begin{aligned}
\frac{1}{\left(q, q a_{1}^{2} ; q\right)_{\infty}} \sum_{k=0}^{\infty} \frac{1}{-} & a_{1}^{2} q^{2 k} \\
1-a_{1}^{2} & \frac{\left(a_{1}^{2} ; q\right)_{k}}{(q ; q)_{k}}(-1)^{k} q^{\left({ }_{2}^{k+1}\right)}\left(a_{1} b q^{k}, b q^{-k} / a_{1} ; p\right)_{\infty} \\
& \times \int_{0}^{\pi} \frac{\left(e^{2 i \theta}, e^{-2 i \theta} ; q\right)_{\infty}\left(a_{1} q^{k+1} e^{i \theta}, a_{1} q^{k+1} e^{-i \theta} ; q\right)_{\infty}}{\left(a_{1} q^{k} e^{i \theta}, a_{1} q^{k} e^{-i \theta} ; q\right)_{\infty} \prod_{j=2}^{5}\left(a_{j} e^{i \theta}, a_{j} e^{-i \theta} ; q\right)_{\infty}} d \theta .
\end{aligned}
$$

The first equality in (5-2) follows from (1-2). The second equality follows from the form of the Nassrallah-Rahman integral stated in [Gasper and Rahman 2004, (6.3.7)] with

$$
f=a_{1} q^{k}
$$

When $p=q$, Theorem 5.1 should reduce to the Nassrallah-Rahman integral (1-2). This is not obvious, so we will indicate how it works. When $p=q$,

$$
(-1)^{k} q^{\left(\begin{array}{c}
k+1 \\
2
\end{array}\right)}\left(a_{1} b q^{k}, b q^{-k} / a_{1} ; p\right)_{\infty}=\left(a_{1} b, b / a_{1} ; q\right)_{\infty} \frac{b^{k}\left(q a_{1} / b ; q\right)_{k}}{a^{k}\left(a_{1} b ; q\right)_{k}} .
$$

We use the second equation in (5-2) and write the ${ }_{8} W_{7}$ as a sum over $j$. With $\ell=j+k$, the left-hand side of (5-2) becomes

$$
\begin{aligned}
& \frac{2 \pi\left(a_{1} b, b / a_{1} ; q\right)_{\infty} \prod_{s=2}^{5}\left(a_{1} a_{2} a_{3} a_{4} a_{5} / a_{s} ; q\right)_{\infty}}{\left(q, a_{1}^{2} a_{2} a_{3} a_{4} a_{5} ; q\right)_{\infty} \prod_{1 \leq r<s \leq 5}\left(a_{r} a_{s} ; q\right)_{\infty}} \\
& \times \sum_{\ell=0}^{\infty} \frac{1-a_{1}^{2} a_{2} a_{3} a_{4} a_{5} q^{2 \ell-1}}{1-a_{1}^{2} a_{2} a_{3} a_{4} a_{5} / q} \frac{\left(a_{2} a_{3} a_{4} a_{5} / q, a_{1}^{2} a_{2} a_{3} a_{4} a_{5} / q ; q\right)_{\ell}}{\left(q, q a_{1}^{2} ; q\right)_{\ell}} q^{\ell} \\
& \times \prod_{r=2}^{5} \frac{\left(a_{1} a_{r} ; q\right)_{\ell}}{\left(a_{1} a_{2} a_{3} a_{4} a_{5} / a_{r} ; q\right)_{\ell}} \\
& \times{ }_{6} W_{5}\left(a_{1}^{2} ; q a_{1} / b, a_{1}^{2} a_{2} a_{3} a_{4} a_{5} q^{\ell-1}, q^{-\ell} ; q, q b / a_{1}^{2} a_{2} a_{3} a_{4} a_{5}\right) .
\end{aligned}
$$

The ${ }_{6} W_{5}$ can be summed by [Gasper and Rahman 2004, (II.20)], and the expression above reduces to the integral evaluation [Gasper and Rahman 2004, (6.3.7)].

The next theorem generalizes the evaluation of the moments of the AskeyWilson weight function.

Theorem 5.2. We have the integral evaluation

$$
\int_{0}^{\pi} \frac{\left(e^{2 i \theta}, e^{-2 i \theta} ; q\right)_{\infty}\left(b e^{i \theta}, b e^{-i \theta} ; p\right)_{n}}{\prod_{j=1}^{4}\left(a_{j} e^{i \theta}, a_{j} e^{-i \theta} ; q\right)_{\infty}} d \theta
$$




$$
\begin{gathered}
=\frac{2 \pi\left(a_{1} a_{2} a_{3} a_{4} ; q\right)_{\infty}}{(q ; q)_{\infty} \prod_{1 \leq j<k \leq 4}\left(a_{j} a_{k} ; q\right)_{\infty}} \frac{\left(a_{1} a_{2}, q a_{1} a_{3}, q a_{1} a_{4} ; q\right)_{n}}{\left(q, q a_{1}^{2}, a_{1} a_{2} a_{3} a_{4} ; q\right)_{n}} \\
\times \sum_{k=0}^{n} \frac{1-a_{1}^{2} q^{2 k}}{1-a_{1}^{2}} \frac{\left(a_{1}^{2}, q^{-n} ; q\right)_{k}}{\left(q, a_{1}^{2} q^{n+1} ; q\right)_{k}}\left(a_{1} b q^{k}, b q^{-k} / a_{1} ; p\right)_{n} \\
\quad \times q^{k(n+1)} \frac{\left(1-a_{1} a_{3}\right)\left(1-a_{1} a_{4}\right)}{\left(1-a_{1} a_{3} q^{k}\right)\left(1-a_{1} a_{4} q^{k}\right)} \\
\quad \times{ }_{4} \phi_{3}\left(\begin{array}{c}
q^{k-n}, q, a_{3} a_{4}, a_{1} q^{k+1} / a_{2} \\
a_{1} a_{3} q^{k+1}, a_{1} a_{4} q^{k+1}, q^{1-n} / a_{1} a_{2}
\end{array} \mid q, q\right) .
\end{gathered}
$$

Proof. Observe that

$$
\begin{aligned}
\left(a b q^{k}\right. & \left., b q^{-k} / a ; p\right)_{n} \\
& =(a b ; p)_{n} \prod_{j=0}^{n-1}\left(1-a p^{-j} / b\right) q^{-k n}\left(-\frac{b}{a}\right)^{n} \prod_{j=0}^{n-1} \frac{\left(a b p^{j} ; q\right)_{k}\left(a q p^{-j} / b ; q\right)_{k}}{\left(a b p^{j} ; q\right)_{k}\left(a p^{-j} / b ; q\right)_{k}} \\
& =(a b, b / a ; p)_{n} q^{-k n} \prod_{j=0}^{n-1} \frac{\left(a b p^{j} ; q\right)_{k}\left(a q p^{-j} / b ; q\right)_{k}}{\left(a p^{-j} / b, a b p^{j} ; q\right)_{k}} .
\end{aligned}
$$

Hence,

$$
\begin{aligned}
\sum_{k=0}^{n} \frac{1-a_{1}^{2} q^{2 k}}{1-a_{1}^{2}} & \frac{\left(a_{1}^{2}, a_{1} e^{i \theta}, a_{1} e^{-i \theta}, q^{-n} ; q\right)_{k}}{\left(q, q a_{1} e^{-i \theta}, q a_{1} e^{i \theta}, a_{1}^{2} q^{n+1} ; q\right)_{k}} q^{k(n+1)}\left(a_{1} b q^{k}, \frac{b q^{-k}}{a_{1}} ; p\right)_{n} \\
= & \left(a_{1} b, b / a_{1} ; p\right)_{n} \sum_{k=0}^{n} \frac{1-a_{1}^{2} q^{2 k}}{1-a_{1}^{2}} \frac{\left(a_{1}^{2}, a_{1} e^{i \theta}, a_{1} e^{-i \theta}, q^{-n} ; q\right)_{k}}{\left(q, q a_{1} e^{i \theta}, q a_{1} e^{-i \theta}, a_{1}^{2} q^{n+1} ; q\right)_{k}} q^{k} \\
& \times \prod_{j=0}^{n-1} \frac{\left(q a_{1} p^{-j} / b, q a_{1} b p^{j} ; q\right)_{k}}{\left(a_{1} b p^{j}, a_{1} p^{-j} / b ; q\right)_{k}} \\
= & \left(a_{1} b, b / a_{1} ; p\right)_{n} \frac{\left(q a_{1}^{2}, q ; q\right)_{n}\left(b e^{i \theta}, b e^{-i \theta} ; p\right)_{n}}{\left(q a_{1} e^{i \theta}, q a_{1} e^{-i \theta} ; q\right)_{n}\left(a_{1} b, b / a_{1} ; p\right)_{n}} \\
= & \frac{\left(q, q a_{1}^{2} ; q\right)_{n}\left(b e^{i \theta}, b e^{-i \theta} ; p\right)_{n}}{\left(q a_{1} e^{i \theta}, q a_{1} e^{-i \theta} ; q\right)_{n}} .
\end{aligned}
$$

So, we have

$$
\begin{aligned}
& \frac{\left(q, q a_{1}^{2} ; q\right)_{n}\left(b e^{i \theta}, b e^{-i \theta} ; p\right)_{n}}{\left(q a_{1} e^{i \theta}, q a_{1} e^{-i \theta} ; q\right)_{n}} \\
& \quad=\sum_{k=0}^{n} \frac{1-a_{1}^{2} q^{2 k}}{1-a_{1}^{2}} \frac{\left(a_{1}^{2}, a_{1} e^{i \theta}, a_{1} e^{-i \theta}, q^{-n} ; q\right)_{k}}{\left(q, q a_{1} e^{-i \theta}, q a_{1} e^{i \theta}, a_{1}^{2} q^{n+1} ; q\right)_{k}} q^{k(n+1)}\left(a_{1} b q^{k}, \frac{b q^{-k}}{a_{1}} ; p\right)_{n} .
\end{aligned}
$$


Therefore,

$$
\begin{array}{r}
\int_{0}^{\pi} \frac{\left(e^{2 i \theta}, e^{-2 i \theta} ; q\right)_{\infty}\left(b e^{i \theta}, b e^{-i \theta} ; p\right)_{n}}{\prod_{j=1}^{4}\left(a_{j} e^{i \theta}, a_{j} e^{-i \theta} ; q\right)_{\infty}} d \theta \\
=\frac{1}{\left(q, q a_{1}^{2} ; q\right)_{n}} \sum_{k=0}^{n} \frac{1-a_{1}^{2} q^{2 k}}{1-a_{1}^{2}} \frac{\left(a_{1}^{2}, q^{-n} ; q\right)_{k}}{\left(q, a_{1}^{2} q^{n+1} ; q\right)_{k}} q^{k(n+1)}\left(a_{1} b q^{k}, \frac{b q^{-k}}{a_{1}} ; p\right)_{n} \\
\times \int_{0}^{\pi} \frac{\left(e^{2 i \theta}, e^{-2 i \theta} ; q\right)_{\infty}}{\left(a_{1} q^{n+1} e^{i \theta}, a_{1} q^{n+1} e^{-i \theta}, a_{1} q^{k} e^{i \theta}, a_{1} q^{k} e^{-i \theta} ; q\right)_{\infty}} \\
\times \frac{\left(a_{1} q^{k+1} e^{i \theta}, a_{1} q^{k+1} e^{-i \theta} ; q\right)_{\infty}}{\prod_{j=2}^{4}\left(a_{j} e^{i \theta}, a_{j} e^{-i \theta} ; q\right)_{\infty}} d \theta
\end{array}
$$

Using [Gasper and Rahman 2004, (6.3.8)] and Watson's formula [Gasper and Rahman 2004, (III.18)], the integral in the equation above becomes

$$
\begin{aligned}
& \frac{2 \pi\left(a_{1} a_{2} a_{3} a_{4} ; q\right)_{\infty}}{(q ; q)_{\infty} \prod_{1 \leq j<k \leq 4}\left(a_{j} a_{k} ; q\right)_{\infty}} \frac{\left(a_{1} a_{2} ; q\right)_{n}\left(a_{1} a_{3}, a_{1} a_{4} ; q\right)_{n+1}}{\left(a_{1} a_{2} a_{3} a_{4} ; q\right)_{n}} \\
& \quad \times \frac{1}{\left(1-a_{1} a_{3} q^{k}\right)\left(1-a_{1} a_{4} q^{k}\right)} 4 \phi_{3}\left(\begin{array}{c}
q^{k-n}, q, a_{3} a_{4}, a_{1} q^{k+1} / a_{2} \\
a_{1} a_{3} q^{k+1}, a_{1} a_{4} q^{k+1}, q^{1-n} / a_{1} a_{2}
\end{array} \mid q, q\right) .
\end{aligned}
$$

This completes the proof.

We give a second proof of (5-3) because it has an idea which may be useful in other cases. The second proof uses the following recent result of [Ismail and Stanton 2010]:

$$
\begin{aligned}
& \frac{\left(q, q a^{2} ; q\right)_{n}}{\left(q a e^{i \theta}, q a e^{-i \theta} ; q\right)_{n}}\left(b e^{i \theta}, b e^{-i \theta} ; p\right)_{n} \\
= & \sum_{k=0}^{n} \frac{1-a^{2} q^{2 k}}{1-a^{2}} \frac{\left(q^{-n}, a^{2}, a e^{i \theta}, a e^{-i \theta} ; q\right)_{k}}{\left(q, a^{2} q^{n+1}, a q e^{i \theta}, a q e^{-i \theta} ; q\right)_{k}} q^{k(1+n)}\left(a b q^{k}, b q^{-k} / a ; p\right)_{n} .
\end{aligned}
$$

Second proof of Theorem 5.2. In view of (5-4), the left-hand side of (5-3) is

$$
\begin{aligned}
& \frac{1}{\left(q, q a_{1}^{2} ; q\right)_{n}} \sum_{k=0}^{n} \frac{1-}{-a_{1}^{2} q^{2 k}}\left.\frac{\left(q^{-n}, a_{1}^{2} ; q\right)_{k}}{1-a_{1}^{2}} q^{k(1+n)}\left(a_{1} b q^{k}, b q^{-k} / a_{1} ; p\right)_{n} q^{n+1} ; q\right)_{k} \\
& \quad \times \int_{0}^{\pi} \frac{\left(e^{2 i \theta}, e^{-2 i \theta} ; q\right)_{\infty}\left(a_{1} q^{k+1} e^{i \theta}, a_{1} q^{k+1} e^{-i \theta}\right)_{n-k}}{\left(a_{1} q^{k} e^{i \theta}, a_{1} q^{k} e^{-i \theta} ; q\right)_{\infty} \prod_{j=2}^{4}\left(a_{j} e^{i \theta}, a_{j} e^{-i \theta} ; q\right)_{\infty}} d \theta .
\end{aligned}
$$


This integral can be evaluated by (3-1) and equals

$$
\begin{aligned}
& \frac{2 \pi\left(a_{1}^{2} q^{2 k+1}, q ; q\right)_{n-k}\left(q^{k} a_{1} a_{2} a_{3} a_{4} ; q\right)_{\infty}}{(q ; q)_{\infty} \prod_{j=2}^{4}\left(q^{k} a_{1} a_{j} ; q\right)_{\infty} \prod_{2 \leq r<s \leq 4}\left(a_{r} a_{s} ; q\right)_{\infty}} \\
& \quad \times{ }_{4} \phi_{3}\left(\begin{array}{c}
q^{k-n}, a_{1} a_{2} q^{k}, a_{1} a_{3} q^{k}, a_{1} a_{4} q^{k} \\
a_{1}^{2} q^{2 k+1}, a_{1} a_{2} a_{3} a_{4} q^{k}, q^{-n}
\end{array} \mid q, q\right)
\end{aligned}
$$

The application of the iterated Sears transformation [Gasper and Rahman 2004, (III.16)] reduces ${ }_{4} \phi_{3}$ to

$$
\frac{\left(a_{1} a_{2} q^{k}, a_{1} a_{3} q^{k+1}, a_{1} a_{4} q^{k+1} ; q\right)_{n-k}}{\left(a_{1}^{2} q^{2 k+1}, a_{1} a_{2} a_{3} a_{4} q^{k}, q ; q\right)_{n-k}}
$$

times the ${ }_{4} \phi_{3}$ in (5-3). Simple manipulations now establish (5-3).

Let $p=1$ and $\zeta=\frac{1}{2}(b+1 / b)$. Then,

$$
\begin{aligned}
& \int_{0}^{\pi} \frac{\left(e^{2 i \theta}, e^{-2 i \theta} ; q\right)_{\infty}}{\prod_{j=1}^{4}\left(a_{j} e^{i \theta}, a_{j} e^{-i \theta} ; q\right)_{\infty}}(\cos \theta-\zeta)^{n} d \theta \\
& =\frac{2 \pi\left(a_{1} a_{2} a_{3} a_{4} ; q\right)_{\infty}}{(q ; q)_{\infty} \prod_{1 \leq j<k \leq 4}\left(a_{j} a_{k} ; q\right)_{\infty}} \frac{\left(a_{1} a_{2}, q a_{1} a_{3}, q a_{1} a_{4} ; q\right)_{n}}{\left(q, q a_{1}^{2}, a_{1} a_{2} a_{3} a_{4} ; q\right)_{n}} \\
& \times \sum_{k=0}^{n} \frac{1-a_{1}^{2} q^{2 k}}{1-a_{1}^{2}} \frac{\left(a_{1}^{2}, q^{-n} ; q\right)_{k}}{\left(q, a_{1}^{2} q^{n+1} ; q\right)_{k}}\left(\frac{1}{2}\left(a_{1} q^{k}+q^{-k} / a_{1}\right)-\zeta\right)^{n} \\
& \times q^{k} \frac{\left(1-a_{1} a_{3}\right)\left(1-a_{1} a_{4}\right)}{\left(1-a_{1} a_{3} q^{k}\right)\left(1-a_{1} a_{4} q^{k}\right)} \\
& \times{ }_{4} \phi_{3}\left(\begin{array}{c|c}
q^{k-n}, q, a_{3} a_{4}, a_{1} q^{k+1} / a_{2} \\
a_{1} a_{3} q^{k+1}, a_{1} a_{4} q^{k+1}, q^{1-n} / a_{1} a_{2}
\end{array} \mid q, q\right) .
\end{aligned}
$$

The special case $\zeta=0$ gives the Askey-Wilson moments

$$
\begin{aligned}
\int_{-1}^{1} W(x ; \mathbf{a}) x^{n} d x= & \frac{\left(a_{1} a_{2}, q a_{1} a_{3}, q a_{1} a_{4} ; q\right)_{n}}{\left(2 a_{1}\right)^{n}\left(q, q a_{1}^{2}, a_{1} a_{2} a_{3} a_{4} ; q\right)_{n}} \\
& \times \sum_{k=0}^{n} \frac{1-a_{1}^{2} q^{2 k}}{1-a_{1}^{2}} \frac{\left(a_{1}^{2}, q^{-n} ; q\right)_{k}}{\left(q, a_{1}^{2} q^{n+1} ; q\right)_{k}}\left(1+a_{1}^{2} q^{2 k}\right)^{n} \\
& \times q^{k(n+1)} \frac{\left(1-a_{1} a_{3}\right)\left(1-a_{1} a_{4}\right)}{\left(1-a_{1} a_{3} q^{k}\right)\left(1-a_{1} a_{4} q^{k}\right)} \\
& \times{ }_{4} \phi_{3}\left(\begin{array}{c}
q^{k-n}, q, a_{3} a_{4}, a_{1} q^{k+1} / a_{2} \\
a_{1} a_{3} q^{k+1}, a_{1} a_{4} q^{k+1}, q^{1-n} / a_{1} a_{2}
\end{array} \mid q, q\right),
\end{aligned}
$$


where $W$ is the normalized weight function

$$
W(x ; \mathbf{a}):=\frac{(q ; q)_{\infty} \prod_{1 \leq r<s \leq 4}\left(a_{r} a_{s} ; q\right)_{\infty}}{2 \pi\left(a_{1} a_{2} a_{3} a_{4} ; q\right)_{\infty}} w(x ; \mathbf{a}) .
$$

The moments of the Askey-Wilson weight functions were first computed in the very interesting paper [Corteel and Williams 2007]. Corteel and Williams used purely combinatorial techniques and showed that the moments of the AskeyWilson weight is a generating function for purely combinatorial objects. The Corteel-Williams formula is very different in nature from our (5-6), and a very interesting but difficult exercise is to show the equivalence of the two results.

\section{The Andrews identities}

We now prove both (1-4) and (1-5) using the ${ }_{5} \phi_{4}$ to ${ }_{12} \phi_{11}$ transformation [Gasper and Rahman 2004, (2.8.4)].

Proof of (1-4). The limiting case $e \rightarrow 0$ of the ${ }_{5} \phi_{4}$ to ${ }_{12} \phi_{11}$ transformation (2.8.4) of [Gasper and Rahman 2004] is

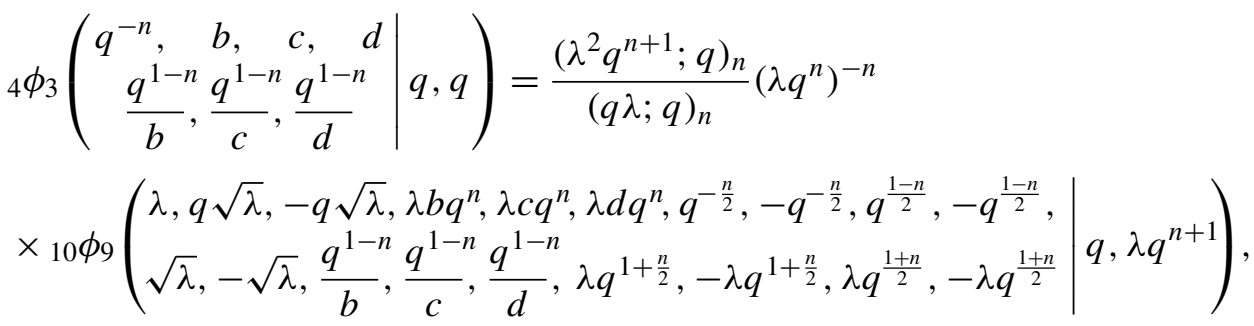

where $b c d \lambda=q^{1-2 n}$. Thus, the ${ }_{4} \phi_{3}$ above is

$$
\begin{array}{r}
\frac{\left(\lambda q^{n}\right)^{-n}}{(\lambda q ; q)_{n}} \sum_{k=0}^{\lfloor n / 2\rfloor} \frac{1-\lambda q^{2 k}}{1-\lambda} \frac{\left(q^{-n} ; q\right)_{2 k}\left(\lambda . \lambda b q^{n}, \lambda c q^{n}, \lambda d q^{n} ; q\right)_{k}}{\left(q, q^{1-n} / b, q^{1-n} / c, q^{1-n} / d ; q\right)_{k}} \\
\quad \times \frac{\left(\lambda^{2} q^{n+1} ; q\right)_{n}}{\left(\lambda^{2} q^{n+1} ; q\right)_{2 k}}\left(\lambda q^{n+1}\right)^{k} \\
=\frac{\left(\lambda q^{n}\right)^{-n}}{(\lambda q ; q)_{n}} \sum_{k=0}^{\lfloor n / 2\rfloor} \frac{1-\lambda q^{2 k}}{1-\lambda} \frac{\left(q^{-n} ; q\right)_{2 k}\left(\lambda . \lambda b q^{n}, \lambda c q^{n}, \lambda d q^{n} ; q\right)_{k}}{\left(q, q^{1-n} / b, q^{1-n} / c, q^{1-n} / d ; q\right)_{k}} \\
\times\left(\lambda^{2} q^{n+1+2 k} ; q\right)_{n-2 k}\left(\lambda q^{n+1}\right)^{k},
\end{array}
$$

since $(a ; q)_{n} /(a ; q)_{j}=\left(a q^{j} ; q\right)_{n-j}$. In the case of (1-4), we replace $q$ by $q^{2}$, then replace $b, c$ and $d$ by $a, b$ and $q^{1-2 n} / a b$, respectively. These choices make $\lambda=q^{1-2 n}$. Hence, the ${ }_{4} \phi_{3}$ in (1-4) transforms to 
(6-1) $\frac{q^{-n}}{\left(q^{3-2 n} ; q^{2}\right)_{n}} \sum_{k=0}^{\lfloor n / 2\rfloor} \frac{1-q^{1-2 n+4 k}}{1-q^{1-2 n}} \frac{\left(q^{1-2 n}, q a, q b, q^{2-2 n} / a b ; q^{2}\right)_{k}}{\left(q^{2}, q^{2-2 n} / a, q^{2-2 n} / b, q a b ; q^{2}\right)_{k}}$

$$
\times q^{3 k}\left(q^{4-2 n+4 k} ; q^{2}\right)_{n-2 k}\left(q^{-2 n} ; q^{2}\right)_{2 k} .
$$

Since $\left(q^{4-2 n+2 k} ; q^{2}\right)_{n-2 k}=q^{-2\left(\begin{array}{c}n-2 k \\ 2\end{array}\right)}\left(-q^{2}\right)^{n-2 k}\left(q^{-2} ; q^{2}\right)_{n-2 k}$ by [Gasper and Rahman 2004, (I.8)], we find that the summand of the series above vanishes, unless $0 \leq n-2 k \leq 1$, which implies that the only nonvanishing term is when $k=\lfloor n / 2\rfloor$. Computing and simplifying this last term gives the right-hand side of (1-4).

Proof of (1-5). The use of the easily verifiable identity

$$
\frac{1-a b q^{-1}}{1-a b^{2} q^{2 n-3}} \frac{\left(b, q^{3-2 n} / a b ; q^{2}\right)_{k}}{\left(q^{4-2 n} / b, a b / q ; q^{2}\right)_{k}}-a b^{2} q^{2 n-3} \frac{\left(b, q^{3-2 n} / a b ; q^{2}\right)_{k}}{\left(q^{2-2 n} / b, q a b ; q^{2}\right)_{k}}
$$

gives

$$
=\frac{\left(b, q^{2-2 n} / a b ; q^{2}\right)_{k}}{\left(q^{4-2 n} / b, q a b ; q^{2}\right)_{k}}
$$

$$
\begin{gathered}
{ }_{4} \phi_{3}\left(\begin{array}{c}
q^{-2 n}, a, b, q^{3-2 n} / a b \\
q^{2-2 n} / a, q^{4-2 n} / b, q a b
\end{array} \mid q^{2}, q^{2}\right) \\
=\frac{1-a b q^{-1}}{1-a b^{2} q^{2 n-3}}{ }_{4} \phi_{3}\left(\begin{array}{c}
q^{-2 n}, a, b, q^{3-2 n} / a b \\
q^{2-2 n} / a, q^{4-2 n} / b, a b / q
\end{array} \mid q^{2}, q^{2}\right) \\
\quad-a b^{2} q^{2 n-3}{ }_{4} \phi_{3}\left(\begin{array}{c}
q^{-2 n}, a, b, q^{3-2 n} / a b \\
q^{2-2 n} / a, q^{2-2 n} / b, q a b
\end{array} \mid q^{2}, q^{2}\right)
\end{gathered}
$$

yielding two balanced and nearly-poised series of the second kind on the righthand side. Now we use the Watson transformation formula [Gasper and Rahman 2004, (III.18)] to transform the right-hand side of into ${ }_{8} \phi_{7}$ series. Thus

$$
\begin{array}{r}
{ }_{4} \phi_{3}\left(\begin{array}{c}
q^{-2 n}, a, b, \frac{q^{3-2 n}}{a b} \\
\frac{q^{2-2 n}}{a}, \frac{q^{4-2 n}}{b}, q a b
\end{array} \mid q^{2}, q^{2}\right)=\frac{1-a b q^{-1}}{1-a b^{2} q^{2 n-3}} \frac{\left(a / q, b / q ; q^{2}\right)_{n}}{\left(1 / q, a b / q ; q^{2}\right)_{n}} \\
\quad \times{ }_{8} \phi_{7}\left(\begin{array}{c}
q^{1-2 n}, q^{-n+5 / 2},-q^{-n+5 / 2}, q^{-2 n}, a, q a, \frac{b}{q}, b \\
\left.q^{-n+1 / 2},-q^{-n+1 / 2}, q^{3}, \frac{q^{3-2 n}}{a}, \frac{q^{2-2 n}}{a}, \frac{q^{4-2 n}}{b}, \frac{q^{3-2 n}}{b} \mid q^{2}, \frac{q^{6-2 n}}{a^{2} b^{2}}\right)
\end{array}\right. \\
-a b^{2} q^{2 n-3} \frac{\left(b / q, a b ; q^{2}\right)_{n}}{\left(1 / q, a ; q^{2}\right)_{n}}{ }_{8} \phi_{7}\left(\begin{array}{c}
q^{1-2 n}, q^{-n+5 / 2},-q^{-n+5 / 2}, q^{-2 n}, b, q b, \frac{q^{2-2 n}}{a b}, \frac{q^{3-2 n}}{a b} \mid q^{2}, a^{2} \\
q^{-n+1 / 2},-q^{-n+1 / 2}, q^{3}, \frac{q^{3-2 n}}{b}, \frac{q^{2-2 n}}{b}, q a b, a b
\end{array}\right) .
\end{array}
$$


The crucial formula to use now is the quadratic transformation formula [Gasper and Rahman 2004, (3.5.10)], that after some simplification, gives

$$
\begin{aligned}
{ }_{4} \phi_{3}\left(\begin{array}{c}
q^{-2 n}, a, b, q^{3-2 n} / a b \\
q^{2-2 n} / a, q^{4-2 n} / b, q a b
\end{array} \mid q^{2}, q^{2}\right) \\
=\frac{\left(q^{2-2 n} ; q\right)_{2 n}}{\left(q^{2-2 n} / a ; q\right)_{2 n}} \frac{\left(a b q^{n-2},-a b q^{n-2} ; q\right)_{n}}{\left(b q^{n-2},-b q^{n-2} ; q\right)_{n}} a^{-2 n} \frac{1-a b q^{-1}}{1-a b^{2} q^{2 n-3}} \\
\quad \times \sum_{k=0}^{n} \frac{1+q^{2-2 n+2 k} / b}{1+q^{2-2 n} / b} \frac{\left(-q^{2-2 n} / b, q^{2-n} / b,-q^{2-n} / b, a ; q\right)_{k}}{\left(q, q^{3-n} / b,-q^{3-n} / b,-q^{3-2 n} / a ; q\right)_{k}} \frac{q^{2 k}\left(q^{-2 n} ; q^{2}\right)_{k}}{a^{k}\left(q^{2-2 n} ; q^{2}\right)_{k}} \\
-a b q^{3 n-3} \frac{\left(b / q, a b, a^{2} ; q^{2}\right)_{n}}{\left(1 / q, a, a^{2} b^{2} q^{2} ; q^{2}\right)_{n}} \frac{\left(q^{2-2 n} ; q\right)_{2 n}}{\left(q^{2-2 n} / b ; q\right)_{2 n}} \\
\quad \times \sum_{k=0}^{n} \frac{1+a b q^{2 k}}{1+a b} \frac{\left(-a b, b, a b q^{n-1},-a b q^{n-1} ; q\right)_{k}}{\left(q,-q a, b, a b q^{n+1},-a b q^{n+1} ; q\right)_{k}} \frac{q^{2 k}\left(q^{-2 n} ; q^{2}\right)_{k}}{b^{k}\left(q^{2-2 n} ; q^{2}\right)_{k}}
\end{aligned}
$$

However, in each of the two series above there is the common factor

$$
\frac{\left(q^{2-2 n} ; q\right)_{2 n}\left(q^{-2 n} ; q^{2}\right)_{k}}{\left(q^{2-2 n} ; q^{2}\right)_{k}}=\left(q^{2-2 n+2 k} ; q^{2}\right)_{n-k}\left(q^{-2 n} ; q^{2}\right)_{k}\left(q^{3-2 n} ; q^{2}\right)_{n},
$$

which vanishes unless $k=n$. So, the only term that survives in each is the one term with $k=n$. Combining the two terms after a lot of messy but straightforward simplifications, we obtain (1-5).

\section{Acknowledgements}

We thank the referee for a careful reading of the manuscript. The research of Mourad Ismail is supported by a grant from King Saud University in Riyadh.

\section{References}

[Andrews 1986] G. E. Andrews, q-series: their development and application in analysis, number theory, combinatorics, physics, and computer algebra, CBMS Regional Conference Series in Mathematics 66, American Mathematical Society, Providence, RI, 1986. MR 88b:11063 Zbl 0594.33001 [Andrews 1987] G. E. Andrews, "Catalan numbers, $q$-Catalan numbers and hypergeometric series", J. Combin. Theory Ser. A 44:2 (1987), 267-273. MR 88f:05015 Zbl 0607.05006

[Andrews 2011] G. E. Andrews, "On Shapiro's Catalan convolution”, Adv. in Appl. Math. 46:1-4 (2011), 15-24. MR 2794010 Zbl 05895437

[Andrews et al. 1999] G. E. Andrews, R. Askey, and R. Roy, Special functions, Encyclopedia of Mathematics and its Applications 71, Cambridge University Press, Cambridge, 1999. MR 2000g: 33001 Zbl 0920.33001

[Askey and Wilson 1985] R. Askey and J. Wilson, Some basic hypergeometric orthogonal polynomials that generalize Jacobi polynomials, Mem. Amer. Math. Soc. 319, American Mathematical Society, Providence, RI, 1985. MR 87a:05023 
[Brown et al. 1996] B. M. Brown, W. D. Evans, and M. E. H. Ismail, "The Askey-Wilson polynomials and $q$-Sturm-Liouville problems", Math. Proc. Cambridge Philos. Soc. 119:1 (1996), 1-16. MR 96j:33012 Zbl 0860.33012

[Corteel and Williams 2007] S. Corteel and L. K. Williams, "Tableaux combinatorics for the asymmetric exclusion process", Adv. in Appl. Math. 39:3 (2007), 293-310. MR 2008g:05220 Zbl 1129. 05057

[Corteel and Williams 2010] S. Corteel and L. K. Williams, "Staircase tableaux, the asymmetric exclusion process, and Askey-Wilson polynomials", Proc. Natl. Acad. Sci. USA 107:15 (2010), 6726-6730. MR 2011k:05268 Zbl 1205.05243

[Erdélyi et al. 1953] A. Erdélyi, W. Magnus, F. Oberhettinger, and F. G. Tricomi, Higher transcendental functions, vol. 2, McGraw-Hill Book Company, New York-Toronto-London, 1953. Based, in part, on notes left by Harry Bateman. MR 15,419i Zbl 0052.29502

[Gasper and Rahman 2004] G. Gasper and M. Rahman, Basic hypergeometric series, 2nd ed., Encycl. of Mathematics and its Applications 96, Cambridge University Press, 2004. MR 2006d:33028 Zbl 1129.33005

[Goldman and Rota 1970] J. Goldman and G.-C. Rota, "On the foundations of combinatorial theory. IV. Finite vector spaces and Eulerian generating functions”, Studies in Appl. Math. 49 (1970), 239258. MR $42 \# 93$

[Ismail 1995] M. E. H. Ismail, "The Askey-Wilson operator and summation theorems", pp. 171178 in Mathematical analysis, wavelets, and signal processing (Cairo, 1994), edited by M. E. H. Ismail et al., Contemp. Math. 190, Amer. Math. Soc., Providence, RI, 1995. MR 96j:33011 Zbl 0839.33010

[Ismail 2009] M. E. H. Ismail, Classical and quantum orthogonal polynomials in one variable, Encycl. of Mathematics and its Applications 98, Cambridge University Press, 2009. Reprint of the 2005 original. MR 2010i:33001 Zbl 1172.42008

[Ismail and Stanton 1998] M. E. H. Ismail and D. Stanton, "More orthogonal polynomials as moments", pp. 377-396 in Mathematical essays in honor of Gian-Carlo Rota (Cambridge, MA, 1996), edited by B. E. Sagan and R. P. Stanley, Progr. Math. 161, Birkhäuser, Boston, MA, 1998. MR 99f: 33011 Zbl 0905.05083

[Ismail and Stanton 2003] M. E. H. Ismail and D. Stanton, " $q$-Taylor theorems, polynomial expansions, and interpolation of entire functions", J. Approx. Theory 123:1 (2003), 125-146. MR 2004g: 30040 Zbl 1035.30025

[Ismail and Stanton 2010] M. E. H. Ismail and D. Stanton, "Some combinatorial and analytical identities", preprint, 2010, available at www.math.umn.edu/ stanton/PAPERS/FuLascoux.pdf.

[Ismail and Zhang 1994] M. E. H. Ismail and R. Zhang, "Diagonalization of certain integral operators", Adv. Math. 109:1 (1994), 1-33. MR 96d:39005 Zbl 0838.33012

[Ismail and Zhang 2005] M. E. H. Ismail and R. Zhang, "New proofs of some $q$-series results", pp. 285-299 in Theory and applications of special functions, edited by M. E. H. Ismail and E. Koelink, Dev. Math. 13, Springer, New York, 2005. MR 2006b:33029 Zbl 1219.33019

[Ismail et al. 1996] M. E. H. Ismail, M. Rahman, and R. Zhang, "Diagonalization of certain integral operators. II”, J. Comput. Appl. Math. 68:1-2 (1996), 163-196. MR 98d:33011 Zbl 0868.33015

[Koekoek and Swarttouw 1998] R. Koekoek and R. F. Swarttouw, "The Askey-scheme of hypergeometric orthogonal polynomials and its $q$-analogues", technical report 98-17, Faculty of Technical Mathematics and Informatics, TU Delft, 1998, available at http://homepage.tudelft.nl/11r49/ documents/as98.pdf. 
[Krattenthaler 1989] C. Krattenthaler, " $q$-analogue of a two variable inverse pair of series with applications to basic double hypergeometric series", Canad. J. Math. 41:4 (1989), 743-768. MR 90g: 33004 Zbl 0667.33005

[Krattenthaler 1996] C. Krattenthaler, "A new matrix inverse”, Proc. Amer. Math. Soc. 124:1 (1996), 47-59. MR 96d:15004 Zbl 0843.15005

[Krattenthaler and Schlosser 1999] C. Krattenthaler and M. Schlosser, "A new multidimensional matrix inverse with applications to multiple $q$-series", Discrete Math. 204:1-3 (1999), 249-279. MR 2000j:33011 Zbl 0936.33011

[Nassrallah and Rahman 1985] B. Nassrallah and M. Rahman, "Projection formulas, a reproducing kernel and a generating function for $q$-Wilson polynomials", SIAM J. Math. Anal. 16:1 (1985), 186-197. MR 87b:33009 Zbl 0564.33009

[Rahman 1987] M. Rahman, "An integral representation and some transformation properties of $q$ Bessel functions", J. Math. Anal. Appl. 125:1 (1987), 58-71. MR 88h:33020 Zbl 0634.33013

[Rainville 1960] E. D. Rainville, Special functions, Macmillan, New York, 1960. MR 21 \#6447 Zbl 0092.06503

[Riordan 1968] J. Riordan, Combinatorial identities, Wiley, New York, 1968. MR 38 \#53 Zbl 0194. 00502

[Rota 1964] G.-C. Rota, "On the foundations of combinatorial theory. I. Theory of Möbius functions", Z. Wahrscheinlichkeitstheorie und Verw. Gebiete 2 (1964), 340-368. MR 30 \#4688

[Slater 1966] L. J. Slater, Generalized hypergeometric functions, Cambridge University Press, 1966. MR 34 \#1570 Zbl 0135.28101

Received June 29, 2010. Revised March 28, 2011.

Mourad E. H. IsMaIL

DEPARTMENT OF MATHEMATICS

City University OF HONG Kong

Tat Chee Avenue

KOWLOON

HONG KONG

and

DEPARTMENT OF MATHEMATICS

KING SAUD UNIVERSITY

RIYADH

SAUdi ARABIA

ismail@math.ucf.edu

MIZAN RAHMAN

DEPARTMENT OF MATHEMATICS

CARLETON UNIVERSITY

OTTAWA, ONTARIO K1S5B6

CANADA

mrahman@math.carleton.ca 


\title{
PACIFIC JOURNAL OF MATHEMATICS
}

\author{
http://www.pjmath.org \\ Founded in 1951 by
}

E. F. Beckenbach (1906-1982) and F. Wolf (1904-1989)

\section{EDITORS}

V. S. Varadarajan (Managing Editor)

Department of Mathematics

University of California

Los Angeles, CA 90095-1555

pacific@math.ucla.edu

Vyjayanthi Chari

Department of Mathematics

University of California

Riverside, CA 92521-0135

chari@math.ucr.edu

\section{Robert Finn}

Department of Mathematics Stanford University

Stanford, CA 94305-2125

finn@math.stanford.edu

Kefeng Liu

Department of Mathematics

University of California

Los Angeles, CA 90095-1555

liu@math.ucla.edu
Darren Long

Department of Mathematics

University of California

Santa Barbara, CA 93106-3080

long@math.ucsb.edu

Jiang-Hua Lu

Department of Mathematics

The University of Hong Kong

Pokfulam Rd., Hong Kong jhlu@maths.hku.hk

Alexander Merkurjev

Department of Mathematics University of California

Los Angeles, CA 90095-1555 merkurev@math.ucla.edu
Sorin Popa

Department of Mathematics

University of California

Los Angeles, CA 90095-1555

popa@math.ucla.edu

Jie Qing

Department of Mathematics

University of California

Santa Cruz, CA 95064

qing@ cats.ucsc.edu

Jonathan Rogawski

Department of Mathematics

University of California

Los Angeles, CA 90095-1555

jonr@math.ucla.edu

\section{PRODUCTION}

pacific@math.berkeley.edu

Silvio Levy, Scientific Editor Matthew Cargo, Senior Production Editor

ACADEMIA SINICA, TAIPEI

CALIFORNIA INST. OF TECHNOLOGY

INST. DE MATEMÁTICA PURA E APLICADA

KEIO UNIVERSITY

MATH. SCIENCES RESEARCH INSTITUTE

NEW MEXICO STATE UNIV.

OREGON STATE UNIV.

\section{SUPPORTING INSTITUTIONS}

STANFORD UNIVERSITY
UNIV. OF BRITISH COLUMBIA
UNIV. OF CALIFORNIA, BERKELEY
UNIV. OF CALIFORNIA, DAVIS
UNIV. OF CALIFORNIA, LOS ANGELES
UNIV. OF CALIFORNIA, RIVERSIDE
UNIV. OF CALIFORNIA, SAN DIEGO
UNIV. OF CALIF., SANTA BARBARA

UNIV. OF CALIF., SANTA CRUZ

UNIV. OF MONTANA

UNIV. OF OREGON

UNIV. OF SOUTHERN CALIFORNIA

UNIV. OF UTAH

UNIV. OF WASHINGTON

WASHINGTON STATE UNIVERSITY

These supporting institutions contribute to the cost of publication of this Journal, but they are not owners or publishers and have no responsibility for its contents or policies.

See inside back cover or www.pjmath.org for submission instructions.

The subscription price for 2011 is US \$420/year for the electronic version, and \$485/year for print and electronic.

Subscriptions, requests for back issues from the last three years and changes of subscribers address should be sent to Pacific Journal of Mathematics, P.O. Box 4163, Berkeley, CA 94704-0163, U.S.A. Prior back issues are obtainable from Periodicals Service Company, 11 Main Street, Germantown, NY 12526-5635. The Pacific Journal of Mathematics is indexed by Mathematical Reviews, Zentralblatt MATH, PASCAL CNRS Index, Referativnyi Zhurnal, Current Mathematical Publications and the Science Citation Index.

The Pacific Journal of Mathematics (ISSN 0030-8730) at the University of California, c/o Department of Mathematics, 969 Evans Hall, Berkeley, CA 94720-3840, is published monthly except July and August. Periodical rate postage paid at Berkeley, CA 94704, and additional mailing offices. POSTMASTER: send address changes to Pacific Journal of Mathematics, P.O. Box 4163, Berkeley, CA 94704-0163.

PJM peer review and production are managed by EditFLOW ${ }^{\mathrm{TM}}$ from Mathematical Sciences Publishers.

PUBLISHED BY PACIFIC JOURNAL OF MATHEMATICS

at the University of California, Berkeley 94720-3840

A NON-PROFIT CORPORATION

Typeset in IATEX

Copyright $(2011$ by Pacific Journal of Mathematics 


\title{
PACIFIC JOURNAL OF MATHEMATICS
}

\author{
Volume $252 \quad$ No. $2 \quad$ August 2011
}

Remarks on a Künneth formula for foliated de Rham cohomology

MÉLANIE BERTELSON

$K$-groups of the quantum homogeneous space ${ }_{q}(n) /_{q}(n-2)$

275

PARTHA SARATHI CHAKRABORTY and S. SUNDAR

A class of irreducible integrable modules for the extended baby TKK algebra

293

Xuewu Chang and ShaObin TAN

Duality properties for quantum groups

SOPHIE CHEMLA

Representations of the category of modules over pointed Hopf algebras over $\mathbb{S}_{3}$ and $\mathrm{S}_{4}$

Agustín García IGLESIAS and Martín Mombelli

$(p, p)$-Galois representations attached to automorphic forms on ${ }_{n}$

EKNATH GHATE and NARASIMHA KUMAR

On intrinsically knotted or completely 3-linked graphs 407

Ryo Hanaki, Ryo NikKUni, Kouki TaNiYama and AKIKo YAMAZAKI

Connection relations and expansions

Mourad E. H. ISMAIL and Mizan Rahman

Characterizing almost Prüfer $v$-multiplication domains in pullbacks

QING LI

Whitney umbrellas and swallowtails

TAKASHI NISHIMURA

The Koszul property as a topological invariant and measure of singularities

HAL SADOFSKY and BRAD SHELTON

A completely positive map associated with a positive map

ERLING STøRMER

Classification of embedded projective manifolds swept out by rational homogeneous varieties of codimension one

KiWAMU Watanabe

Note on the relations in the tautological ring of $\mu_{g}$ 